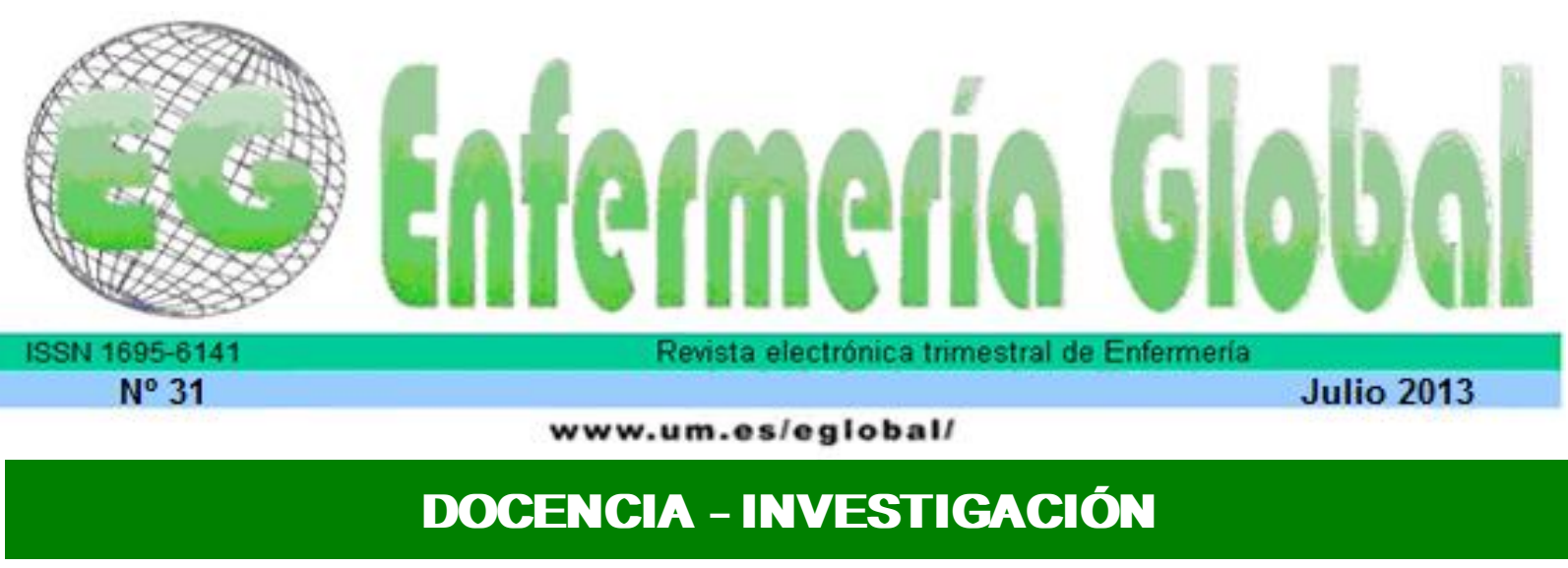

\title{
Salud familiar general: familias de mujeres en lactancia materna, Villamaría (Caldas) Colombia
}

Familiar general health: breast feeding women's families Villamaria (Caldas)

\section{*Betancurth Loaiza, Diana Paola **Amaya Rey, María Consuelo del Pilar}

*Facultad de Ciencias para la Salud, Manizales, Universidad de Caldas. E-mail: vlogomes@terra.com.br ***Facultad de Enfermería. Programa de Doctorado en Enfermería, Universidad Nacional de Colombia.

Palabras clave: Salud de la familia; riesgo; enfermería; investigación; lactancia materna; Promoción de la Salud.

Keywords: Family Health; Nursing; Research; Breast Feeding; Health Promotion; Risk; Primary Health Care.

\section{RESUMEN}

Fundamento: La Salud Familiar (SF) consta de dos dimensiones cotidianas: la organización familiar y la satisfacción con la organización.

Objetivos: 1. Caracterizar la Salud Familiar Total de las familias de mujeres en lactancia materna (MLM) desde los dos componentes. 2. Encontrar la relación/Predicción entre Salud Familiar Total (SFT) y Riesgo Familiar Total (RFT) de las familias.

Métodos: Cuantitativo, descriptivo, correlacional, transversal, realizado con las familias de Mujeres en Lactancia Materna en servicio ambulatorio de Villamaría (Caldas) Colombia durante el año 2011.

Muestra: Aleatoria de 76 familias, seleccionadas con un error admisible de 2 unidades de registro, y un nivel de confianza de .95 evaluadas con el instrumento de "Salud Familiar General ISFG: GEN-21 y Riesgo Familiar Total RFT: 5-33" (1).

Resultados: El análisis de la Salud Familiar mostró familias muy saludables 83 por ciento (63) y familias saludables con 17 por ciento. Más de la tercera parte se percibieron como organizadas y muy organizadas. El 97 por ciento de las familias se perciben entre satisfechas y muy satisfechas con la organización familiar.

Conclusiones: Las familias estudiadas con Mujeres en Lactancia materna son saludables. Estas requieren de la promoción de la salud desde enfermería y demás profesionales, por los resultados de Salud Familiar General que son una fortaleza, debe mantenerse permanentemente, como potencial de la salud de las familias durante la lactancia materna y los estadios posteriores de la trayectoria. Se confirma que la relación entre Salud familiar y Riesgo Familiar Total es inversa y significativa. 


\section{ABSTRACT}

Background: Familial health $(\mathrm{FH})$ consists of two daily dimensions: familial organization and also the satisfaction with the organization.

Objectives: 1. Make the overall maternal women's feeding familial health characterization (MWF) from two listed constituents. 2: To find the relationship /prediction between on the Total Familial Health (TFH) and also Total Familial Risk (TFR) on the families.

Methods: Quantitative, descriptive, with correlation, and also transversal, making to the Maternal Women's feeding families within outdoor service of Villamaria (Caldas) Colombia on the year 2011.

Sample: Randomizing over 76 families selecting by an allowable mistake with 2 overall records' units and with a confidence level of .95 families testing by the instrument "Salud Familiar General ISFG: GEN-21 and Riesgo Familiar Total RFT: 5-33(1)".

Results: The Health Family analysis showed 83 percent pretty healthy families (63) and healthy families with 17 percent. More than the third part of them was seen as organized and also very organized families. 97 percent of the families are drawing as satisfied and very satisfied families, within the familial organization.

Conclusions: The families that were studied with maternal women's feeding were finding healthy. Those families are requiring the health promoting programs from nursing and also other professional fields, by general familiar health results that are a fortress, must be permanently holding, as a health potential field during maternal women's feeding and the subsequent studies over the trajectory. The inverted relationship between Familial Health and Total Familial Risk was confirmed.

\section{INTRODUCCIÓN}

La familia es la célula básica de la sociedad, de ahí la importancia que tiene el estudio de los eventos, estadíos y problemas que con mayor frecuencia pudieran ocasionar crisis en cuanto a la organización cotidiana. Según Veliz "la familia es un elemento fundamental para el desempeño exitoso de la lactancia materna" ${ }^{(1)}$.

La presente investigación buscó desde la salud familiar entendida como la organización de la familia y la satisfacción con esa organización, obtener información específica para el cuidado de enfermería familiar sobre las diferentes dimensiones de proceso de la familia: mantenimiento, cohesión individuación y cambio que son los elementos que componen las categorías que determinan la salud familiar desde organización y satisfacción familiar (Friedemann, 1995, citada por Amaya) ${ }^{(2)}$ dentro de los diferentes niveles de promoción y prevención. Así, la intervención familiar en Atención Primaria en Salud (APS) va desde la promoción de la salud hasta los diferentes niveles de prevención para garantizar Salud Pública de los grupos de familias con MLM.

La acción con la familia va desde el extremo de hacerla sana y que permanezca saludable hasta abordar los problemas de salud de sus miembros individuales y del grupo. Según Louro ${ }^{(3)}$ la atención a los problemas de la familia en su micro-espacio social contribuye a mejorar la situación de salud familiar y comunitaria, por tanto, la identificación de sus características y necesidades es fundamental para la estrategia de la Atención Primaria en Salud y la materialización concreta de la equidad en salud, es así como el conocimiento de la situación de salud familiar es un aspecto importante de la atención integral a la salud en el nivel primario. 
Así, la exigencia para enfermería y los equipos de salud consiste en ayudar al grupo familiar como un todo a cumplir adecuadamente las funciones fundamentales como familia para que se mantenga, se cohesione, logre la individuación de cada uno de sus integrantes y se transforme con los cambios internos y externos requeridos de la trayectoria. Además construir un entorno o ambiente congruente al desarrollo humano individual, que les permita crecer y desarrollarse respetando su dignidad y de acuerdo con sus expectativas y necesidades, la familia busca mantener su equilibrio conforme evoluciona. Su condición de salud depende de una adaptación exitosa a los desafíos cambiantes de la vida familiar y al desarrollo de sus miembros, propios del ciclo vital familiar ${ }^{(4)}$.

El trabajo de enfermería con la familia cada vez exige más conocimientos científicos que permitan entender el interior de los sistemas familiares, como lo dicen Sinche y Suárez ${ }^{(4)}$ aunque el Equipo de Atención Integral de Salud se esfuerce por responder a las necesidades de las familias, el desconocimiento de elementos clave para atender estos requerimientos puede llevar a una inefectiva, o incluso contraproducente intervención.

De acuerdo con este concepto es fundamental que el cuerpo de enfermería vaya más allá de las acciones empíricas y obtenga herramientas realmente valiosas para el conocimiento de la familia a través de la valoración, al respecto Núñez y Barros ${ }^{(5)}$ refieren como en todos los lugares donde enfermería trabaja su centro de interés es la familia: su salud, su capacidad de crecimiento, los cuidados de sí misma y su manera de contribuir a la comunidad.

Dentro de la práctica de la lactancia materna, el núcleo familiar ejerce funciones sociales fundamentadas por relaciones de intimidad, afecto y solidaridad. En la dinámica familiar se encuentran algunas reservas que permiten a las mujeres articularse o desarticularse frente a su vivencia diaria de la lactancia. Estas reservas son consideradas desde las físicas, hasta aquellas que incluyen recursos psíquicos y socioculturales de los miembros de la familia ${ }^{(6)}$. Por tanto debe existir un ambiente familiar favorable, sobre todo en aquellas madres que enfrentan el proceso de lactancia materna por primera vez ${ }^{(1)}$, pues las alteraciones de la vida familiar son capaces de provocar alteración emocional, desequilibrio y descompensación del estado de salud ${ }^{(3)}$ y abandono de la lactancia materna.

Partiendo de esta revisión, el presente estudio se trazó como objetivo principal caracterizar la salud familiar total de las familias de mujeres en lactancia materna desde las dos dimensiones que la evalúan, la escala de Organización Sistémica y Grado de Satisfacción que plantea el instrumento. Como objetivo secundario se propuso encontrar la relación entre RFT y SF de las familias de las MLM.

Entendiéndose la Escala de Salud Familiar Total como la congruencia de la familia en su interior, un constructo elaborado a partir de la evaluación de la Escala de Organización Sistémica la cual presenta ítemes que traducen las operaciones de la familia en la vida cotidiana, de acuerdo con los factores de proceso de cohesión, cambio, individuación y mantenimiento del sistema (Friedemann, 1995) y la Escala de Satisfacción relacionada con la apreciación subjetiva de satisfacción y de conformidad con los patrones habituales de organización de la familia ${ }^{(1)}$. 


\section{MATERIAL Y MÉTODOS}

\section{Muestra y muestreo}

La muestra se fundó sobre el comportamiento estadístico de los resultados de la aplicación del instrumento ISF: GEN-21 y RFT: 5-33. A efectos de conocer el comportamiento estadístico preliminar se determinó una muestra inicial conformada por 60 familias de mujeres lactantes, elegidas aleatoriamente en el servicio de APS, consulta externa del Hospital San Antonio (Programa de Crecimiento y Desarrollo) planeada y ejecutada con el propósito de captar información preliminar, a manera de insumo, para efectuar el cálculo de varios tamaños de muestra acordes con niveles elegibles de error máximo admisible y de confianza de .95.

\section{Muestreo}

Para efecto de contar con una muestra con la mayor holgura posible, se admitió una desviación estándar de 8,8382 y se asumió para población infinita. Con estas consideraciones, el tamaño de la muestra aleatoria simple para el estudio, se calculó mediante la expresión usual para estimar promedios. La expresión algebraica para dicho cálculo fue:

$n=\frac{\left(\mathbf{l} z_{1-\frac{\alpha}{2}}\right) \mathbf{J}^{2} \hat{\theta}^{2}}{e^{2}}$

donde 100 por ciento (1- $\alpha)$ corresponde al nivel de confianza, expresado porcentualmente; ${ }^{z_{1-\frac{\alpha}{2}}}$ el percentil correspondiente, de una distribución normal estándar; $\hat{\sigma}$ la estimación de la desviación estándar en la muestra piloto y e error máximo admisible en la estimación del promedio de la dimensión.

Atendiendo a la viabilidad de identificación y entrevista a la mujer lactante o el miembro familiar seleccionado de su grupo familiar, se optó por una muestra aleatoria simple de tamaño igual a 76 familias, correspondiente a un error máximo admisible de 2 y un nivel de confianza de .95. Se hizo también la prueba piloto a un grupo de 9 familias de mujeres lactantes con el objeto de estimar el tiempo promedio necesario, permitir la familiarización con el instrumento, y determinar la precisión, la forma de formular y consignar las respuestas en el instrumento.

\section{Criterios de inclusión}

Para seleccionar las familias fueron tenidos en cuenta los siguientes criterios ${ }^{(2)}$ :

A). Familias con mujeres de cualquier edad que se encontraran en periodo de lactancia y tuvieran un/a hijo/a con edad comprendida entre un día y doce meses.

B) MLM que acudieran a los servicios de salud del programa de Crecimiento y Desarrollo del servicio de APS de Villamaría y que fueran de estrato socio-económico 1, 2 ó 3. C). El informante familiar debía tener por lo menos 15 años de edad, haber convivido por lo menos durante un año con la familia y contar con capacidad física y mental para responder una entrevista. 
Técnicas y procedimientos de recolección de información: previo a la recolección se contó con la autorización del uso de los instrumentos y el entrenamiento para el diligenciamiento por parte de la autora del mismo ${ }^{(2)}$. La información fue recolectada, en la consulta externa del hospital de Villamaría, durante los meses de marzo y abril de 2011.

El instrumento Salud familiar General ISF: GEN -21 ${ }^{(1)}$ aplicado tiene una validez y confiabilidad sustentada para ser utilizado en la práctica y la investigación. Los coeficientes Alfa de Cronbach, tanto para la escala de Organización Sistémica (.86), como para la escala de Satisfacción (.92) dan razón de una elevada homogeneidad y dan cuenta de la consistencia interna satisfactoria de cada una de las escalas. Los coeficientes Alfa de los factores oscilaron entre .43 y .81 en la escala de Organización Sistémica y entre .53 y .87 en la escala de Satisfacción. El instrumento Salud Familiar General: ISF: GEN-21 está compuesto por tres secciones: la primera contiene los datos de identificación, tipificación y composición de la familia, aunque no representa puntaje alguno para el instrumento; la segunda contiene el libreto de la aplicación que debe ser leído por el entrevistador al participante, mientras que la tercera parte está compuesta por 21 ítems que puntúan en dos escalas de tipo Likert, una para el componente de organización sistémica y otra para el de satisfacción ${ }^{(2) .}$

\section{Puntuación de las escalas de SF}

Como se anunció, la SF está definida y puntúa en términos de las dos escalas: En la escala de Organización Sistémica: puntajes estandarizados (PE) altos (superiores a PE: 65) ubican a una familia en la vía de un sistema familiar muy organizado mientras que puntajes bajos (inferiores a PE: 45) indican que la familia está en un sistema poco organizado. En la escala de satisfacción: puntajes altos (superiores a PE: 65), sitúan a una familia en la vía de alto grado y satisfacción, mientras que puntajes bajos (inferiores a PE: 45) indican una familia poco satisfecha con el sistema. En cuanto a la escala de Salud Familiar Total: puntajes altos (superiores a pe: 64) ubican a una familia en vía de alto grado de salud familiar, mientras que puntajes bajos (inferiores a pe: 45) señalan una familia poco saludable.

El instrumento de Riesgo Familiar Total (RFT: 5-33) ${ }^{(7)}$ fue la segunda herramienta utilizada. Aplicado a las 76 familias, cuenta con los datos de validez y confiabilidad sustentada para ser utilizado en la práctica y la investigación. La confiabilidad fue estimada por la autora de los instrumentos ${ }^{(7)}$ mediante el coeficiente de consistencia Kuder-Richardson \# 20 (KR20) (Kuder et Richardson, 1937). El instrumento de Riesgo Familiar Total consta de cinco secciones principales, las cuatro primeras identifican y describen aspectos para el conocimiento, la tipificación de familia y la historia de la trayectoria, y la puntuación se obtiene de los ítems que puntúan en la sección quinta compuesta por 33 ítems de Factores de Riesgo con los que se evalúa en conjunto Riesgo Familiar Total, así: Condiciones psicoafectivas, Servicios y prácticas de salud, Condiciones de vivienda y vecindario, Situación socioeconómica y Manejo de menores. Según Amaya el puntaje total del instrumento RFT: 5-33, se define en tres niveles de calificación del Riesgo Familiar, en términos de escala, como familias en riesgo bajo, familias amenazadas y familias con riesgo alto según los puntajes brutos y estandarizados ${ }^{(2)}$. 


\section{Análisis de datos}

Al terminar la recolección, se realizó la imputación de datos para el control de calidad en el diligenciamiento de los instrumentos, luego fueron digitados en una base de datos en Excel. El análisis estadístico se hizo con el programa SPSS v 15.0 (SPSS inc., Chicago IL.) y SAS v 9.0.

\section{RESULTADOS}

Se dan a conocer los resultados obtenidos producto de la aplicación del instrumento Salud familiar General ISF: GEN -21 del instrumento Riesgo Familiar Total y Riesgo Familiar Total: 5-33 donde fueron estudiadas 76 familias de MLM residentes en el municipio de Villamaría con un total de 367 miembros, con un mínimo de 2 integrantes y un máximo de 11, el promedio fue de 4.83 miembros por familia.

\section{Resultados Salud Familiar General}

Los resultados obtenidos muestran la distribución de las familias de las tres subcategorías, familias muy saludables $(83 \% n=63)$ y familias saludables $(17 \% n=13)$, no se encontraron familias que se consideraran poco saludables.

La figura 1 muestra que más de la tercera parte de la muestra se percibió entre familias organizadas y muy organizadas, predominando ésta última categoría.

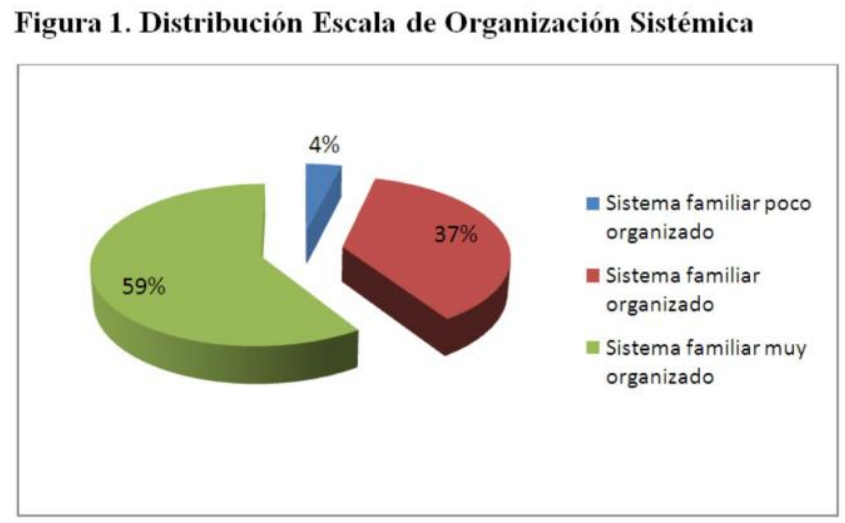

Fuente: Resultado según instrumento ISF: GEN 21. Villamaría-Caldas

La figura 2 muestra que casi la totalidad de las familias se perciben entre satisfechas y muy satisfechas con un total de 97 por ciento $(n=74)$. 
Figura 2. Distribución Escala de Satisfacción.

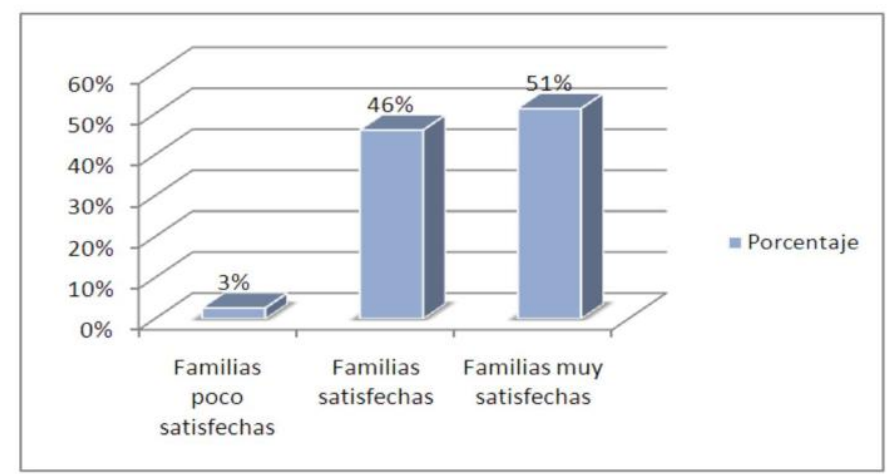

Fuente: Resultado según instrumento ISF: GEN 21. Villamaría-Caldas.

Las familias investigadas presentan un alto porcentaje de cohesión y cambio como se muestra en la tabla I; con respecto a cohesión, 96 por ciento de los grupos familiares se consideraron "organizados" entendiendo la cohesión como la relación entre los miembros de la familia; en lo relacionado con el cambio, 72 por ciento de los grupos familiares se consideraron "poco organizados".

Tabla I. Factores de Cohesión y Cambio en las Familias de mujeres lactantes

\begin{tabular}{llllll}
\hline \multirow{2}{*}{ Dimensión } & Calificación & \multicolumn{2}{l}{ Escala de Acuerdos } & \multicolumn{2}{l}{ Escala de Satisfacción } \\
& & Frec & $\%$ & Frec & $\%$ \\
\hline \multirow{2}{*}{ Cohesión } & Poco organizado & 3 & 4 & 10 & 13 \\
& Organizado & 73 & 96 & 66 & 87 \\
& Poco organizado & 4 & 5 & 8 & 11 \\
Cambio & Organizado & 72 & 95 & 68 & 89 \\
\hline
\end{tabular}

Fuente: Resultado según instrumento ISF: GEN 21. Villamaría-Caldas

\section{Resultados de Riesgo Familiar Total}

La distribución de la frecuencia del RFT por puntuaciones estandarizadas muestra la clasificación en riesgo alto $(3 \%)$, riesgo bajo $(35 \%)$ y el riesgo para las familias amenazadas (62\%). Esta última categoría tiene un predominio importante con respecto a las demás; llama la atención el bajo porcentaje para familias de alto riesgo. En cuanto a cada uno de los cinco factores (RFT), la dimensión más afectada corresponde a las dimensiones psicoafectivas en familias amenazadas junto con la de servicios y prácticas en salud en las familias en alto riesgo.

Las correlaciones/ Predicciones de Salud Familiar Total y todas las posibilidades de Riesgo familiar.

\section{Predicción del RFT a partir del comportamiento del ISF: GEN21}

En la tabla II se presentan los parámetros asociados a la recta de regresión. En primer lugar, se observa que el parámetro asociado a la variable Salud Familiar Total es significativamente diferente de cero ( $p \_v a l o r=0.006$ ) lo que muestra una asociación 
entre estos dos pares de variables. En conclusión, el grado de Salud Familiar Total es un predictor del Riesgo Familiar Total pero quizá no sea el único ni el mejor. Por otra parte la correlación entre la Salud Familiar Total SFT y Riesgo Familiar Total, RFT son inversamente proporcionales y Significativos.

Tabla II. Predicción del RFT a partir del comportamiento del GEN21

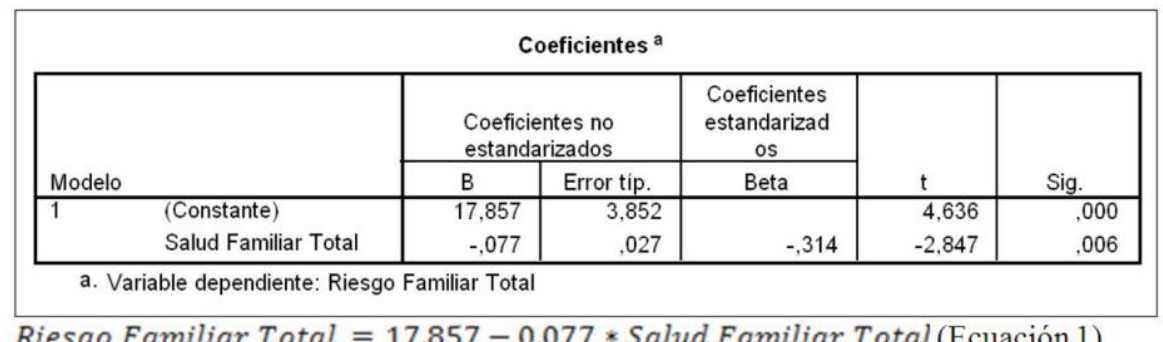

Fuente: Resultado según instrumento ISF: GEN 21. Villamaría-Caldas

\section{Predicción del GEN21 a partir del comportamiento del RFT}

En la tabla III se presentan los parámetros asociados a la recta de regresión. Al igual que el caso anterior, se observa que el parámetro asociado a la variable Riesgo Familiar Total es significativamente diferente de cero ( $p \_$valor $=0.006$ ) lo que muestra, como previamente se sabía ${ }^{(7)}$, asociación entre estos dos pares de variables. En la Ecuación 2 se presenta la recta de regresión de predicción ajustada para el grado de Salud Familiar Total en función del Riesgo Familiar Total. Al igual que la ecuación 1, el Riesgo Familiar Total es un buen predictor del grado de Salud Familiar Total pero éste no es el único y quizá tampoco el mejor ( $R$ cuadrado de 0.099).

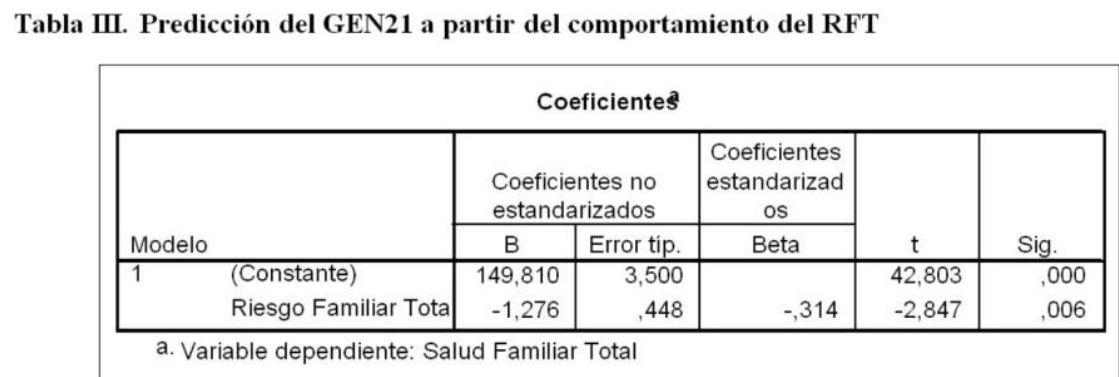

Salud Familiar Total $=149.81-1.276 *$ Riesgo Familiar Total $($ Ecuación 2$)$

Fuente: Resultado según instrumento ISF: GEN 21. Villamaría-Caldas

Correlaciones de Organización familiar y Satisfacción familiar con Resultados del Riesgo Familiar Total.

A partir de diagramas de dispersión entre los pares de variables de interés y luego estimar el coeficiente de correlación de Pearson para cada uno de los diagramas presentados y los resultados expuestos en la tabla IV en efecto, se observa una mayor correlación (aunque leve) entre el comportamiento de la organización sistémica y el Riesgo Familiar Total (-0.356) que entre esta última variable y la escala de satisfacción (-0.240). No obstante, ambos coeficientes de correlación son significativamente diferentes de cero lo que indica que existe asociación estadística entre estos pares de variables. 
Tabla IV. Correlaciones de Organización familiar y Satisfacción familiar con Resultados del Riesgo Familiar Total

\begin{tabular}{|c|c|c|}
\hline Variables de Interés & $\begin{array}{l}\text { Coeficiente de } \\
\text { Correlación de } \\
\text { Pearson }\end{array}$ & P_valor \\
\hline $\begin{array}{l}\text { Organización Sistémica } \\
\text { vs Riesgo Familiar } \\
\text { Total }\end{array}$ & -0.356 & 0.002 \\
\hline $\begin{array}{l}\text { Escala de Satisfacción } \\
\text { vs Riesgo Familiar } \\
\text { Total }\end{array}$ & -0.240 & 0.037 \\
\hline
\end{tabular}

Fuente: Resultado según instrumento ISF: GEN 21. Villamaría-Caldas

\section{DISCUSIÓN}

Un porcentaje alto de familias refleja que la percepción de estar aparentemente saludables es muy alta, por encima de lo encontrado por Pérez ${ }^{\left({ }^{9}\right)}$ con familias de adolescentes gestantes después de la valoración con el instrumento ISF: GEN21, el cual obtuvo un promedio de 54.1 por ciento y coherente con los resultados para Caldas, que es uno de los departamentos que clasifica la salud como excelente, muy buena o buena según los datos de la Encuesta Nacional de Salud 2010 (ENDS 2010) $)^{(10)}$. Cabe destacar que el estudio se efectuó entre habitantes del sector urbano, tipo de población con mayor tendencia a percibir su salud como excelente", muy buena" y buena" que quienes viven en la zona rural (ENDS 2010) (10).

Las familias de las mujeres que lactan se consideraron satisfechas con lo que la familia es y lo que hace en la organización interna cotidiana. M. Yaque ${ }^{(11)}$, dio a conocer que debe existir un ambiente familiar favorable, sobre todo en aquellas madres que enfrentan esta experiencia por primera vez, el apoyo de la familia es esencial para que disminuya la ansiedad de la madre que es un factor importante y frecuente en éstas. Veliz y colaboradores ${ }^{(1)}$ en un estudio cubano con mujeres lactantes, concluyeron que el funcionamiento familiar y el apoyo familiar resultaron importantes para el mantenimiento de la lactancia materna exclusiva.

Llama la atención el hecho de que a pesar de pertenecer a estratos de ingresos bajos y medio-bajos, las familias se consideran en un alto porcentaje muy saludables, lo que puede considerarse como fortalezas, las cuales ponen de manifiesto puntos de partida para el análisis no solo de riesgos sino también de potencialidades con el objetivo de mejorar la salud.

\section{CONCLUSIONES}

En conclusión todas las familias (100\%) refirieron percibirse entre saludables y muy saludables cuando se les evaluó la Salud Familiar General (SFG), mientras que frente al Riesgo Familiar Total (RFT) el 62 por ciento de las familias fue ubicado como amenazadas (o de riesgo medio), esto permite identificar claramente la diferencia en que el riesgo es un sistema de abordaje diferente al de salud familiar. Las familias pueden tener problemas asociados al Riesgo Familiar Total que refleja el contexto y la situación de la familia en su interior y exterior y la Salud Familiar General su dinámica interna, lo que quiere decir que no necesariamente el bajo riesgo implica que puede ser calificada en mejor condición la salud familiar. 


\section{Agradecimientos}

Al hospital San Antonio del municipio de Villamaría (Caldas) por facilitar las condiciones para la realización del estudio. a las familias por la participación en este proceso investigativo, a la Facultad de Enfermería de la Universidad Nacional de Colombia y la Facultad de Ciencias para la Salud de la Universidad de Caldas.

\section{REFERENCIAS BIBLIOGRÁFICAS}

1. Veliz JA, Sanabria G, Gálvez AM, Sanabria J, Fernández Z. El período de lactancia materna, su relación con el tipo de familia, funcionamiento y apoyo familiar. Revista

CTMA Avances. 2007; 9(2): 3-6. Disponible en: http://www.ciget.pinar.cu/Revista/No.2007-

2/Art\%EDculos/Publicacion3\%20con\%20resumen\%20traducido.pdf

2. Amaya P. instrumento de salud familiar isf: gen-21. Manual. aspectos teóricos, psicométricos, de estandarización y de aplicación. Santa Fe de Bogotá: Universidad Nacional de Colombia; Unibiblos. 2004; 70.

3. Louro Bernal I, Pría Barros MC. Alternativas metodológicas para la estratificación de familias según situación de salud familiar. Rev Cubana Med Gen Integr [revista en Internet]. 2008 Dic [citado 2012 mayo 20]; 24(4). Disponible en: http://scielo.sld.cu/pdf/mgi/v24n4/mgi06408.pdf

4. Sinche E. y Suárez M. RAMPA, 2006; 1(1):38-47. Disponible en: http://www.idefiperu.org/RAMNRO1/RAMPA\%20V1N1\%20Parte2.pdf

5. Núñez G, Barros Z. Evaluación de la salud de las familias del barrio don Atilio de la ciudad de Salto, aplicando instrumento de Isabel Louro. Revista Uruguaya de Enfermería, nov. 2009; 4 (2): 36-44. Disponible en: http://164.73.124.24/rue/sitio/num8/8_art05_nunez.pdf

6. Spanó AM, Guerreiro MC, Bistafa MJ, Azevedo F. Women's social space and the reference for breastfeeding practice. Rev. Latino-Am. Enfermagem [serial on the Internet] 2007 Apr [cited 2011 Sep 3];15(2):230-238. Available from: http://dx.doi.org/10.1590/S0104-11692007000200007

7. Amaya Rey P. Instrumento Riesgo Familiar Total RTF 5-33 Manual: Aspectos teóricos conceptuales y análisis de la información. Santa Fe de Bogotá: Universidad Nacional de Colombia; Unibiblos; 2004;77.

8. Friedemann ML. The framework of systemic organization. A conceptual approach to families and Nursing. United States of America: Sage publications; 1995; 4-16.

9. Pérez, B. El cuidado de enfermería en familia: un reto para el siglo XXI. Caracterización de las familias con adolescentes gestantes. Bogotá: Revista Aquichan. 2003; 3(1): 21-31. Disponible en: http://aquichan.unisabana.edu.co/index.php/aquichan/article/view/31/61

10. Profamilia. Encuesta Nacional de Demografía y Salud. ENDS. Percepción del estado de salud de la población. Afiliación, Percepción y Uso de Servicios del SGSSS. Cap. 14.1 pp. 399-439. Disponible en: http://www.profamilia.org.co/encuestas/Profamilia/Profamilia/images/stories/PDFcapitulos/Capitulo-14.pdf

11. Yaque M, Castillo E, Praena Crespo M, Sancho C, Fernández A, Herrera $C$ et al. Factores relacionados con el inicio de la lactancia materna en nuestro medio: diferencias entre cuatro zonas básicas de salud. Rev Pediatr Aten Primaria. 2000; 2(6): 231-239. 
๑ COPYRIGHT Servicio de Publicaciones - Universidad de Murcia 